\title{
Inverse problems for the Schrödinger equations with time-dependent electromagnetic potentials and the Aharonov-Bohm effect.
}

\author{
G.Eskin, Department of Mathematics, UCLA, \\ Los Angeles, CA 90095-1555, USA. E-mail: eskin@math.ucla.edu
}

July 30, 2018

\begin{abstract}
We consider the inverse boundary value problem for the Schrödinger operator with time-dependent electromagnetic potentials in domains with obstacles. We extend the resuls of the author's works [E1], [E2], [E3] to the case of time-dependent potentials. We relate our results to the Aharonov-Bohm effect caused by magnetic and electric fluxes.
\end{abstract}

\section{Introduction.}

Let $\Omega_{0}$ be a smooth simply-conneted domain in $\mathbf{R}^{n}$ not necessarily bounded. Let $D \subset \Omega_{0} \times[0, T]$ be a domain with the following properties :

Denote $D_{t_{0}}=D \cap\left\{t=t_{0}\right\}$. We assume that $D_{t_{0}}=\Omega_{0} \backslash \cup_{j=1}^{m} \overline{\Omega_{j}\left(t_{0}\right)}$ where $\Omega_{j}\left(t_{0}\right)$ are a piece-wise smooth nonintersecting domains, $1 \leq j \leq m$. We assume that $\Omega^{\prime}\left(t_{0}\right)=\cup_{j=1}^{m} \Omega_{j}\left(t_{0}\right)$ depends smoothly on $t_{0} \in[0, T]$. We do not assume that $\Omega_{j}\left(t_{0}\right)$ are bounded.

Finally we assume that the normal to $\partial D \backslash\left(D_{0} \cup D_{T}\right)$ in $\mathbf{R}^{n+1}$ is not parallel to the $t$-axis for any $t \in[0, T]$.

Consider the Schrödinger equation with time-dependent electromagnetic potentials in $D$ : 
$(1.1)$

$i \frac{\partial u(x, t)}{\partial t}=H u \stackrel{\text { def }}{=} \sum_{j=1}^{n}\left(-i \frac{\partial}{\partial x_{j}}-A_{j}(x, t)\right)^{2} u(x, t)+V(x, t) u(x, t), \quad(x, t) \in D$,

with zero initial conditions

$$
u(x, 0)=0, \quad(x, 0) \in D_{0},
$$

and the Dirichlet boundary conditions

$$
\begin{array}{r}
\left.u\right|_{\partial \Omega_{0} \times(0, T)}=f, \\
\left.u\right|_{\partial \Omega^{\prime}\left(t_{0}\right)}=0, \quad \forall t_{0} \in[0, T] .
\end{array}
$$

We assume that $A(x, t), V(x, t)$ are smooth in $D$ with compact support. Without loss of generality we can assume that $A(x, t)=0, V(x, t)=0$ near $\partial \Omega_{0} \times[0, T]$ (see Remark 3.1). Let $\Lambda$ be the Dirichlet-to-Neumann (D-to-N) operator on $\partial \Omega_{0} \times[0, T]$, i.e.

$$
\Lambda f=\frac{\partial u}{\partial \nu}-\left.i(A \cdot \nu) u\right|_{\partial \Omega_{0} \times(0, T)},
$$

where $f$ is the same as in $(1.3)$. Denote by $G(D)$ the group of $C^{\infty}(\bar{D})$ functions $c(x, t)$ such that $c(x, t) \neq 0$ in $\bar{D}$ and denote by $G_{0}(D)$ the subgroup of $G(D)$ such that $c(x, t)=1$ on $\partial \Omega_{0} \times[0, T]$. We say that the electromagnetic potentials $(A(x, t), V(x, t))$ and $\left(A^{\prime}(x, t), V^{\prime}(x, t)\right)$ are gauge equivalent if there exists $c(x, t) \in G(D)$ such that

$$
\begin{array}{r}
A_{j}^{\prime}(x, t)=A_{j}(x, t)+i c^{-1}(x, t) \frac{\partial c}{\partial x_{j}}, \quad 1 \leq j \leq n, \\
V^{\prime}(x, t)=V(x, t)-i c^{-1}(x, t) \frac{\partial c}{\partial t} .
\end{array}
$$

Note that if $\left(i \frac{\partial}{\partial t}-H\right) u=0$ and

$$
u^{\prime}=c^{-1} u
$$

then $\left(i \frac{\partial}{\partial t}-H^{\prime}\right) u^{\prime}=0$, where $H^{\prime}$ is the operator with potentials $A^{\prime}(x, t), V^{\prime}(x, t)$. The group $G(D)$ is called the gauge group and (1.6) is called the gauge transformation. When $A(x, t)$ and $V(x, t)$ are real-valued it is natural to consider 
only $c(x, t)$ such that $|c(x, t)|=1$. Also when $D$ is simply connected any $c(x, t) \in G(D)$ has a form $c(x, t)=e^{i \varphi(x, t)}$, where $\varphi(x, t) \in C^{\infty}(\bar{D})$.

We say that the D-to-N operators $\Lambda, \Lambda^{\prime}$ corresponding to the Schrödinger operators $i \frac{\partial}{\partial t}-H, i \frac{\partial}{\partial t}-H^{\prime}$ are gauge equivalent if there exists $c(x, t) \in G(D)$ such that

$$
\Lambda^{\prime}=c_{0}^{-1} \Lambda c_{0} \quad \text { on } \partial \Omega_{0} \times[0, T]
$$

where $c_{0}$ is the restriction of $c(x, t)$ to $\partial \Omega_{0} \times[0, T]$.

We shall introduce gauge invariant boundary data on $\partial \Omega_{0} \times(0, T)$ (c.f. [E1]). Let $u(x, t)$ be a solution of $i \frac{\partial u}{\partial t}-H u=0$ in $D, u(x, 0)=0$.

Let $|u(x, t)|^{2}$ be te probability density and

$$
S(x, t)=\Im\left(\frac{\partial u(x, t)}{\partial x}-i A(x, t) u(x, t)\right) \overline{u(x, t)}
$$

be the probability current. We define the boundary data of $u(x, t)$ on $\partial \Omega_{0} \times$ $(0, T)$ as

$$
\left.|u(x, t)|^{2}\right|_{\partial \Omega_{0} \times(0, T)}=f_{1},\left.\quad \frac{\partial}{\partial \nu}|u(x, t)|^{2}\right|_{\partial \Omega_{0} \times(0, T)}=f_{2},\left.\quad S(x, t)\right|_{\partial \Omega_{0} \times(0, T)}=f_{3},
$$

where $\frac{\partial}{\partial \nu}$ is the normal derivative. Note that the probability density and the probability current are gauge invariant.

The following proposition was proven in [E1]:

Proposition 1.1. Let $\left(i \frac{\partial}{\partial t}-H\right) u=0$ and $\left(i \frac{\partial}{\partial t}-H^{\prime}\right) u^{\prime}=0$ be two Schrödinger equations in $D, u(x, 0)=u^{\prime}(x, 0)=0,\left.u(x, t)\right|_{\partial \Omega^{\prime}(t)}=\left.u^{\prime}\right|_{\partial \Omega^{\prime}(t)}=0$ for all $t \in[0, T]$. Then the $D$-to- $N$ operators $\Lambda$ and $\Lambda^{\prime}$ are gauge equivalent on $\partial \Omega_{0} \times(0, T)$ if and only if for any solution $u$ there exists $u^{\prime}$ such that the gauge invariant boundary data (1.8) of $u(x, t)$ and $u^{\prime}(x, t)$ are equal.

This paper is a completion of works [E1], [E2], [E3] on the inverse problems for the stationary Schrödinger equation in a situation where the AharonovBohm effect caused by magnetic fluxes holds. Here we extend these results to the time-dependent Schrödinger equation with time-dependent electromagnetic potentials in the cases when the Aharonov-Bohm effect holds caused by both the magnetic and electric fluxes.

Consider two Schrödinger operators $i \frac{\partial}{\partial t}-H^{(p)}, p=1,2$, with timedependent electromagnetic potentials $\left(A^{(p)}(x, t), V^{(p)}(x, t)\right), p=1,2$, in the domain $D$ described above. 
Theorem 1.1. Assume that $n \geq 2$ and the domain $D$ is such that for any $t_{0} \in[0, T] \quad D_{t_{0}}$ satisfies the following condition (c.f. [E2], page 287): All $\Omega_{j}\left(t_{0}\right)$ are convex, $1 \leq j \leq m$, and for each point $x_{0} \in D_{t_{0}}$ there exists a two-dimensional plane $\Pi \subset \mathbf{R}^{n}$ such that $\Pi$ intersects at most one domain $\Omega_{j}\left(t_{0}\right), 1 \leq j \leq m$. Let $i \frac{\partial}{\partial t}-H_{j}, j=1,2$, be two Schrödinger operators in $D$ and $\Lambda^{(j)}, j=1,2$, be the corresponding $D$-to- $N$ operator on $\partial \Omega_{0} \times(0, T)$. If $\Lambda^{(1)}$ and $\Lambda^{(2)}$ are gauge equivalent on $\partial \Omega_{0} \times(0, T)$ then the electromagnetic potentials $A^{(1)}(x, t), V^{(1)}(x, t)$ and $A^{(2)}(x, t), V^{(2)}(x, t)$ are gauge equialent in D.

Theorem 1.1 generalized the result of [E2]. Note that when $n=2$ the domain $D_{t_{0}}$ contain at most one convex $\Omega_{j}\left(t_{0}\right)$.

The following theorem generalizing the result of [E3] treats the case when $D_{t_{0}}$ contains more than one obstacle $\Omega_{j}\left(t_{0}\right)$ :

Theorem 1.2. Assume that $n=2$ and that for each $t_{0} \in[0, T]$ domains $\Omega_{j}\left(t_{0}\right), 1 \leq j \leq m$, are piece-wise smooth and convex. Suppose also that there is no trapping broken rays (more exactly, the conditions a), b) in [E3], page $150 \%$, are satisfied). If D-to- $N$ operators $\Lambda^{(1)}$ and $\Lambda^{(2)}$ are gauge equivalent on $\partial \Omega_{0} \times\left(0, T_{0}\right)$, then the electromagnetic potentials $A^{(1)}(x, t), V^{(1)}(x, t)$ and $A^{(2)}(x, t), V^{(2)}(x, t)$ are gauge equivalent on $D$.

Inverse problems and Aharonov-Bohm effect were considered in $[\mathrm{N}],[\mathrm{W}]$. General inverse problems for the stationary Schrödinger equations can be reduced to the inverse problems for the hyperbolic equations with the timeindependent coefficients (see [B], [KKL], [E6] and additional references there). Hyperbolic equations approach is very powerful. However it does not apply to the Schreödinger equations with time-dependent potentials.

The plan of the paper is the following: In $\S 2$ we prove Theorems 1.1 and 1.2. In $\S 3$ we consider the Schrödinger operators with time-dependent Yang-Mills potentials. In $\S 4$ we discuss the Aharonov-Bohm effect (see [AB], [WY], [OP]) in the situations covered by Theorems 1.1 and 1.2. Note that the Aharonov-Bohm effect is caused by the magnetic and electric fluxes, and the cause of electric fluxes appears only when potentials are time-dependent. 


\section{The proof of Theorems 1.1 and 1.2 .}

We shall start with the construction of geometric optics solutions of (1.1). We are looking for a solution in the form

$$
u_{N 0}=e^{-i k^{2} t+i k(x \cdot \omega)} a_{0}^{(N)}
$$

where $a_{0}^{(N)}=\sum_{p=0}^{N} \frac{a_{p 0}(x, t, \omega)}{(i k)^{p}},|\omega|=1, k$ is a large parameter. Substituting $u_{N 0}$ in (1.1) we obtain

$$
k^{2} a_{0}^{(N)}+i \frac{\partial a_{0}^{(N)}}{\partial t}=\left(-i \frac{\partial}{\partial x}+k \omega-A(x, t)\right)^{2} a_{0}^{(N)}+V(x, t) a_{0}^{(N)} .
$$

Equating the equal power of $k$ we get

$$
\begin{gathered}
\omega \cdot\left(-i \frac{\partial}{\partial x}-A\right) a_{00}=0 \\
2 \omega \cdot\left(-i \frac{\partial}{\partial x}-A\right) a_{p 0}=\left(i \frac{\partial}{\partial t}-H\right) a_{p-1,0}, \quad p \geq 1 .
\end{gathered}
$$

To solve (2.2) denote $s=\omega \cdot x, \tau_{j}=\left(\omega_{\perp j}, x\right), 1 \leq j \leq n-1$, where $\omega_{\perp j}, 1 \leq j \leq n-1$, is a basis in an orthogonal complement to $\omega$ in $\mathbf{R}^{n}$. We have $\frac{\partial a_{00}}{\partial s}-i(A \cdot \omega) a_{00}(s, \tau, t)=0$, where $\tau=\left(\tau_{1}, \ldots, \tau_{n-1}\right)$. Therefore we can take

$$
a_{00}(s, \tau, t)=\chi_{1}(t) \chi_{2}(\tau) \exp \left(i \int_{s_{0}}^{s} A\left(\tau+s^{\prime} \omega\right) \cdot \omega d s^{\prime}\right)
$$

where $\chi_{1}(t)=\frac{1}{\sqrt{\varepsilon}} \chi_{0}\left(\frac{t-t_{0}}{\varepsilon}\right), \chi_{2}(\tau)=\frac{1}{\varepsilon^{\frac{n-1}{2}}} \Pi_{j=1}^{n-1} \chi_{0}\left(\frac{\tau_{j}-\tau_{0 j}}{\varepsilon}\right), \chi_{0} \in C_{0}^{\infty}\left(\mathbf{R}^{1}\right), \chi_{0}(t)=$ 0 for $|t|>1, \int_{-\infty}^{\infty} \chi_{0}^{2}(t) d t=1$.

We solve (2.3) prescribing the initial condition

$$
a_{p 0}\left(s_{0}, \tau, t\right)=0, \quad 1 \leq p \leq N
$$

Here $s_{0}, \tau_{0}, t_{0}$ are such that the point $\left(x_{0}^{(0)}=s_{0} \omega+\sum_{j=1}^{n-1} \tau_{0 j} \omega_{\perp j}, t_{0}\right)$ does not belong to $\Omega_{0} \times[0, T]$. We have

$$
\left(i \frac{\partial}{\partial t}-H\right) u_{N 0}=f_{N 0}
$$


where $\hat{f}_{N 0}=\frac{e^{-i k^{2} t+i k(\omega \cdot x)}}{(i k)^{N}}\left(i \frac{\partial}{\partial t}-H\right) a_{N 0}$.

Until now we assume that the ray $x=\left(s-s_{0}\right) \omega+x_{0}^{(0)}$ does not meet the obstacles. In the case when this ray meets an obstacle we have to consider a broken ray that reflects at $\partial \Omega_{j}\left(t_{0}\right), 1 \leq j \leq m$ (c.f. [E3], pages 149899). More precisely, we say that $\gamma=\gamma_{0} \cup \gamma_{1} \cup \ldots \cup \gamma_{r}$ is a broken ray with legs $\gamma_{0}, \gamma_{1}, \ldots, \gamma_{r}$ if $\gamma_{0}$ is the ray $x=x_{0}^{(0)}+\left(s-s_{0}\right) \omega, s_{0} \leq s \leq s_{1}$, $\gamma_{0}$ hits $\partial \Omega^{\prime}\left(t_{0}\right)=\cup_{j=1}^{m} \partial \Omega_{j}\left(t_{0}\right)$ at some point $x_{0}^{(1)}=x_{0}^{(0)}+\left(s_{1}-s_{0}\right) \omega \in \bar{\partial} \Omega^{\prime}\left(t_{0}\right), \gamma_{1}$ is the reflected ray. We assume that $x_{0}^{(1)}$ is not a tangential point of reflection. Then

$$
\theta_{1}=\theta_{0}-2\left(n_{1}\left(x_{0}^{(1)}, t_{0}\right) \cdot \theta_{0}\right) n_{1}\left(x_{0}^{(1)}, t_{0}\right)
$$

where $\theta_{0}=\omega$ is the direction of $\gamma_{0}, \theta_{1}$ is the direction of $\gamma_{1}, n\left(x_{0}^{(1)}, t_{0}\right)$ is the outward unit normal to $\partial \Omega^{\prime}\left(t_{0}\right)$ at the point $x_{0}^{(1)}$. Analoguosly, $\gamma_{2}, \ldots, \gamma_{r-1}$ have nontangential points of reflection on $\partial \Omega^{\prime}\left(t_{0}\right)$ and $\gamma_{r}$ ends on $\partial \Omega_{0} \times\{t=$ $\left.t_{0}\right\}$.

We associate with broken ray $\gamma$ the following geometric optics solution (c.f. [E3], page 1499):

$$
u_{N}=\sum_{j=0}^{r} \sum_{p=0}^{N} \frac{a_{p j}(x, t, \omega)}{(i k)^{p}} e^{-i k^{2} t+i k \psi_{j}(x, t, \omega)},
$$

where $\psi_{0}(x, t, \omega)=x \cdot \omega, a_{0 p}(x, t, \omega)$ are the same as in (2.2), (2.3), (2.4), (2.5), eiconals $\psi_{j}(x, t, \omega)$ satisfy the equations

$$
\begin{gathered}
\left|\frac{\partial \psi_{j}(x, t, \omega)}{\partial x}\right|=1, \\
\left.\psi_{j}(x, t, \omega)\right|_{\partial \Omega^{\prime}(t)}=\left.\psi_{j+1}(x, t, \omega)\right|_{\partial \Omega^{\prime}(t)}, \\
\frac{\partial \psi_{j+1}\left(x_{0}^{(j+1)}, t_{0}, \omega\right)}{\partial x}=\theta_{j+1}, \quad 0 \leq j \leq r-1,
\end{gathered}
$$

where $x_{0}^{(j+1)}$ is the point of reflection of $\gamma_{j}$ at $\partial \Omega^{\prime}\left(t_{0}\right)$ and $\theta_{j+1}$ is the direction of $\gamma_{j+1}$. We assume that $\gamma_{j}, 1 \leq j \leq r$, do not contain caustic points. Then the eiconals $\psi_{j}, 1 \leq j \leq r$, exist. In particular, this is true when $\Omega_{j}(t)$ are convex. Functions $a_{p j}$ satisfy the following equations:

$$
2 \frac{\partial a_{p j}}{\partial x} \cdot \frac{\partial \psi_{j}}{\partial x}+\Delta \psi_{j} a_{p j}-2 i A(x, t) \cdot \frac{\partial \psi_{j}}{\partial x} a_{p j}=f_{p j}(x, t, \omega)+i \frac{\partial \psi_{j}}{\partial t} a_{p j}, p \geq 0
$$


where $f_{0 j}=0, f_{p j}$ depends on $a_{0 j}, \ldots, a_{p-1, j}$. When $\Omega^{\prime}(t)$ is independent of $t$ then $\psi_{j}, j \geq 1$ is also independent of $t$ but $\psi_{j}(x, t, \omega)$ depends on $t$ when $\Omega^{\prime}(t)$ depends on $t$. We impose the following conditions on $a_{p j}$ :

$$
\left.a_{p j}\right|_{\partial \Omega^{\prime}(t)}=-\left.a_{p, j+1}\right|_{\partial \Omega^{\prime}(t)}, \quad 0 \leq j \leq r-1 .
$$

This conditions imply that

$$
\left.u_{N}\right|_{\partial \Omega^{\prime}(t)}=0 .
$$

Substituting (2.7) into (1.1) we get

$$
\left(i \frac{\partial}{\partial t}-H\right) u_{N}=f_{N}
$$

where $f_{N}=\sum_{j=0}^{r} \frac{f_{N j}}{(i k)^{N}} e^{i k \psi_{j}-i k^{2} t} f_{N j}(x, t, \omega)$ are smooth.

In order to complete the construction of the geometric optics solutions we need the following lemma:

Lemma 2.1. Consider the initial-value problem

$$
\begin{array}{r}
\left(i \frac{\partial}{\partial t}-H\right) w=f \quad \text { in } D, \\
\left.w\right|_{\partial D_{t}}=0, \quad \forall t \in(0, T), \\
w(x, 0)=w_{0}(x), \quad(x, 0) \in D_{0} .
\end{array}
$$

Suppose that $\Omega^{\prime}(t)$ is a smooth domain in $\mathbf{R}^{n}$ for $\forall t \in[0, T]$. Then for any $f \in C^{1}\left([0, T], L^{2}\left(D_{t}\right)\right)$ and any $w_{0} \in H^{2}\left(D_{0}\right) \cap \stackrel{\circ}{H^{1}}\left(D_{0}\right)$ there exists a unique $w \in C\left([0, T], H^{1}\left(D_{t}\right) \cap H^{2}\left(D_{t}\right)\right) \cap C^{1}\left([0, T], L^{2}\left(D_{t}\right)\right)$.

Proof: We assume that $A(x, t)$ are real but $V(x, t)$ can be complexvalued.

Make change of variables:

$$
t=t, x^{\prime}=\varphi(x, t), \quad \varphi(x, t)=\left(\varphi_{1}(x, t), \ldots, \varphi_{n}(x, t)\right)
$$

in $\bar{D}$ such that $\varphi(x, t)=x$ on $\partial \Omega_{0} \times[0, T]$ and $\varphi\left(\Omega_{j}(t), t\right)=\Omega_{j}(0), \forall t \in[0, T]$. Denote $\hat{u}\left(x^{\prime}, t\right)=u(x, t)$, where $x^{\prime}=\varphi(x, t)$. Then $u_{t}(x, t)=\hat{u}_{t}+\hat{u}_{x^{\prime}}\left(x^{\prime}, t\right)$. $\varphi_{t}(x, t), u_{x}(x, t)=u_{x^{\prime}} \frac{\partial \varphi}{\partial x}$, etc. 
Therefore (2.15) has the following form in $\left(x^{\prime}, t\right)$ coordinates:

$$
\begin{aligned}
i \frac{\partial \hat{u}}{\partial t} & =\hat{H}_{0} \hat{u}+\hat{H}_{1} \hat{u}+\hat{f}, \quad\left(x^{\prime}, t\right) \in \hat{D} \\
\left.\hat{u}\right|_{\partial \hat{\Omega} \times(0, T)} & =0, \quad \hat{u}\left(x^{\prime}, 0\right)=\hat{u}_{0}\left(x^{\prime}\right), x^{\prime} \in \hat{\Omega},
\end{aligned}
$$

where

$$
\begin{gathered}
\hat{H}_{0}=\frac{1}{\sqrt{g\left(x^{\prime}, t\right)}} \sum_{j, k=1}^{n}\left(-i \frac{\partial}{\partial x_{j}^{\prime}}-\hat{A}_{j}\left(x^{\prime}, t\right)\right) \sqrt{g} g^{j k}\left(x^{\prime}, t\right)\left(-i \frac{\partial}{\partial x_{k}^{\prime}}-\hat{A}_{k}\right), \\
H_{1}=\hat{V}\left(x^{\prime}, t\right)-i \varphi_{t} \cdot \hat{u}_{x^{\prime}} \\
g^{j k}\left(x^{\prime}, t\right)=\sum_{p=1}^{n} \frac{\partial \varphi_{j}}{\partial x_{p}} \frac{\partial \varphi_{k}}{\partial x_{p}}, \quad g\left(x^{\prime}, t\right)=\operatorname{det}\left\|g^{j k}\right\|^{-1} .
\end{gathered}
$$

Note $\hat{D}=\hat{\Omega} \times(0, T], \quad \hat{\Omega}=\Omega_{0} \backslash \bar{\Omega}^{\prime}$, where $\Omega^{\prime}=\cup_{j=1}^{m} \Omega_{j}(0)$.

Multiplying (2.17) by $\sqrt{g\left(x^{\prime}, t\right)} \hat{u}\left(x^{\prime}, t\right)$ and integrating over $\hat{\Omega}$ we get

$$
\left(i \frac{\partial \hat{u}}{\partial t}, \hat{u}\right)_{g}=\left(\hat{H}_{0} \hat{u} . \hat{u}\right)_{g}+\left(\hat{H}_{1} u+f, \hat{u}\right)_{g}
$$

where $\left(v_{1}, v_{2}\right)_{g}$ is the $L_{2}$ inner product in $\hat{\Omega}$ with the weight $\sqrt{\hat{g}}$. Take the imaginary part of (2.18) and integrate in $t$ from 0 to $t$. Since $\hat{H}_{0}$ is self-adjoint and since

$$
\int_{\hat{\Omega}} i \sum_{j} \varphi_{j t} \frac{\partial \hat{u}}{\partial x_{j}} \sqrt{g} \hat{u} d x^{\prime}=-\int_{\hat{\Omega}} i \sum_{j} \varphi_{j t} \sqrt{g} \hat{u} \frac{\overline{\partial \hat{u}}}{\partial x_{j}} d x^{\prime}+\int_{\hat{\Omega}} b\left(x^{\prime}, t\right)|\hat{u}|^{2} d x^{\prime}
$$

for some $b\left(x^{\prime}, t\right)$, we get

$$
\begin{array}{r}
\int_{\hat{\Omega}} \sqrt{g\left(x^{\prime}, t\right)}\left|\hat{u}\left(x^{\prime}, t\right)\right|^{2} d x^{\prime}-\int_{\hat{\Omega}} \sqrt{g\left(x^{\prime}, 0\right)}\left|\hat{u}_{0}\left(x^{\prime}, 0\right)\right|^{2} d x^{\prime} \\
\leq C \int_{0}^{t}\|\hat{u}\|_{0}^{2} d t^{\prime}+C \int_{0}^{t}\|\hat{f}\|_{0}\|\hat{u}\|_{0} d t^{\prime}
\end{array}
$$

where \|\|$_{0}$ is the $L_{2}$ norm in $\hat{\Omega}$.

It follows from (2.20) that

$$
\max _{[0, T]}\|\hat{u}(\cdot, t)\|_{0}^{2} \leq C\left\|\hat{u}\left(x^{\prime}, 0\right)\right\|_{0}^{2}+C \int_{0}^{T}\|\hat{f}(\cdot, t)\|_{0}^{2} d t^{\prime}
$$


Changing from the beginning in (1.1) $u$ to $e^{i \lambda t} u$ we can assume that $\Re V(x, t)$ is large. Since $\left.\hat{u}\right|_{\partial \hat{\Omega} \times(0, T)}=0$ and $\hat{H}_{0}+\hat{H}_{1}$ is elliptic for each $t \in[0, T]$ we get from (2.17), by the standard elliptic theory:

$$
\|\hat{u}\|_{2} \leq C\left\|\frac{\partial \hat{u}}{\partial t}\right\|_{0}+C\|\hat{f}\|_{0}
$$

where $\|\hat{u}\|_{2}$ is the Sobolev norm in $\hat{\Omega}$.

Differentiate (2.17) in $t$ :

$$
i \frac{\partial^{2} \hat{u}}{\partial t^{2}}=\hat{H}_{0} \frac{\partial \hat{u}}{\partial t}+\hat{H}_{1} \frac{\partial \hat{u}}{\partial t}+\hat{H}^{\prime} \hat{u}+\frac{\partial \hat{f}}{\partial t}
$$

where $\hat{H}^{\prime}$ is a differenial operator in $x$ of order at most 2 . Note that $\left.\frac{\partial \hat{u}}{\partial t}\right|_{\partial \hat{\Omega} \times[0, T]}=0$ since $\left.\hat{u}\right|_{\partial \hat{\Omega} \times[0, T]}=0$. Multiplying $(2.23)$ by $\sqrt{g} \frac{\partial \hat{\partial}}{\partial t}$, integrating over $\hat{\Omega} \times(0, t)$ and taking the imaginary part we get, as in (2.20):

$$
\int_{\hat{\Omega}} \sqrt{g}\left|\frac{\partial \hat{u}}{\partial t}\right|^{2} d x^{\prime}-\int_{\hat{\Omega}} \sqrt{g\left(x^{\prime}, 0\right)}\left|\frac{\partial \hat{u}\left(x^{\prime}, 0\right)}{\partial t}\right|^{2} d x^{\prime}
$$

$$
\leq C \int_{0}^{t}\left\|\frac{\partial \hat{u}}{\partial t}\right\|_{0}^{2} d t^{\prime}+C \int_{0}^{t}\|\hat{u}\|_{2}\left\|\frac{\partial \hat{u}}{\partial t}\right\|_{0} d t^{\prime}+C \int_{0}^{t}\left\|\frac{\partial \hat{f}}{\partial t}\right\|_{0}\left\|\frac{\partial \hat{u}}{\partial t}\right\|_{0} d t^{\prime} .
$$

Using (2.22) we get from (2.24)

$$
\max _{[0, T]}\left\|\frac{\partial \hat{u}}{\partial t}\right\|_{0}^{2} \leq C\left\|\frac{\partial \hat{u}(\cdot, 0)}{\partial t}\right\|_{0}^{2}+C \int_{0}^{T}\left(\|\hat{f}\|_{0}^{2}+\left\|\frac{\partial \hat{f}}{\partial t}\right\|_{0}^{2}\right) d t^{\prime} .
$$

It follows from (2.17) that

$$
\left\|\frac{\partial \hat{u}\left(x^{\prime}, 0\right)}{\partial t}\right\|_{0} \leq C\left\|\hat{u}_{0}\left(x^{\prime}, 0\right)\right\|_{2}+\left\|\hat{f}\left(x^{\prime}, 0\right)\right\|_{0}
$$

Combining (2.21), (2.22), (2.25), (2.26) we get

$$
\max _{[0, T]}\|\hat{u}\|_{2}^{2}+\max _{[0, T]}\left\|\frac{\partial \hat{u}}{\partial t}\right\|_{0}^{2} \leq C\left\|\hat{u}_{0}\right\|_{2}^{2}+C \max _{[0, T]}\|\hat{f}\|_{0}^{2}+C \int_{0}^{T}\left\|\frac{\partial \hat{f}}{\partial t}\right\|_{0}^{2} d t^{\prime}
$$

We proved estimate (2.27) assuming that the solution $\hat{u}$ exists. To prove the existence we shall use the parabolic regularization. 
Consider the parabolic equation:

$$
(i-\varepsilon) \frac{\partial u_{\varepsilon}}{\partial t}=\hat{H}_{0} u \varepsilon+\hat{H}_{1} u_{\varepsilon}+\hat{f}, \quad \varepsilon>0,
$$

with the same boundary and initial conditions as in (2.17):

$$
\left.u_{\varepsilon}\right|_{\partial \hat{\Omega} \times(0, T)}=0, \quad u_{\varepsilon}\left(x^{\prime}, 0\right)=\hat{u}_{0}\left(x^{\prime}\right),
$$

By the parabolic theory there exists a solution of (2.28), (2.29) belonging to the same space as $\hat{u}$. Note that the estimate (2.27) holds for $u_{\varepsilon}$ with constants in.dependent of $\varepsilon>0$. Taking the weak limit of a subsequence $\varepsilon_{k} \rightarrow 0$ we get the existence of $\hat{w}$ satisfying (2.17). Note that in the original coordinates $x=\varphi^{-1}\left(x^{\prime}, t\right)$ the estimate (2.27) still holds.

Remark 2.1. In the case of several obstacles we require that $\Omega_{j}(t), 1 \leq$ $j \leq m$, are convex sets having corners. In this case the estimates (2.22) does not hold and one has a weaker estimate:

$$
\|\hat{u}\|_{1} \leq C\left\|\frac{\partial \hat{u}}{\partial t}\right\|_{0}+C\|\hat{f}\|_{0} .
$$

To prove the existence of the solution of (2.17) in this case we assume for simplicity that obstacles $\Omega_{j}(t)$ move as rigid bodies, i.e. the change of variables (2.16) has the form $t=t, \varphi(x, t)=x+\varphi_{j}(t), \varphi_{j}(0)=0$, near $\Omega_{j}(t), 1 \leq j \leq m$. Then $g^{j k}\left(x^{\prime}, t\right)=\delta_{j k}$ near $\Omega^{\prime}$ and the differential operator $\hat{H}^{\prime}$ has the order 1 near $\Omega^{\prime}$. Therefore (2.24) holds with $\|\hat{u}\|_{2}$ replaced by $\|\hat{u}\|_{1}+\|\chi u\|_{2}$ where $\chi=0$ near $\Omega^{\prime}$. Using the elliptic estimate of the form (2.22) for $\chi \hat{u}$ and the estimate (2.30) we get that (2.25) holds in the case of corners too.

Using the parabolic regulation as in (2.28) we get that there exists a unique solution of (2.15) satisfying the estimate (2.27) with $\|\hat{u}\|_{2}$ replaced by $\|\hat{u}\|_{1}$.

By Lemma 2.1 and Remark 2.1 there exists $u^{(N+1)} \in C\left([0, T], H^{1}\left(D_{t}\right)\right)$ such that

$$
\begin{array}{r}
\left(i \frac{\partial}{\partial t}-H\right) u^{(N+1)}=-f_{N} \quad \text { in } D, \\
\left.u^{(N+1)}\right|_{\partial \Omega_{j}^{\prime}(t)}=0, \quad 1 \leq j \leq m, \quad \forall t \in[0, T], \\
u^{(N+1)}(x, 0)=0,\left.\quad u^{(N+1)}\right|_{\partial \Omega_{0} \times(0, T)}=0,
\end{array}
$$


where $f_{N}$ is the same as in (2.14). Then $\left\|u^{(N+1)}\right\|_{1}+\left\|\frac{\partial u^{(N+1)}}{\partial t}\right\|_{0} \leq C k^{-N+1}$ for $\forall t \in[0, T]$. Therefore $u=u_{N}+u^{(N+1)}$ solves

$$
\begin{aligned}
\left(i \frac{\partial}{\partial t}-H\right) u=0, & (x, t) \in D,\left.\quad u\right|_{\partial \Omega_{0} \times(0, T)}=\left.u_{N}\right|_{\partial \Omega_{0} \times(0, T)}, \\
\left.u\right|_{\partial \Omega^{\prime}(t)}=0, & \forall t \in[0, T], \quad u\left(x^{\prime}, 0\right)=0, \quad\left(x^{\prime}, 0\right) \in D_{0} .
\end{aligned}
$$

Now we shall use the Green's formula. Suppose we are given two timedependent Schrödinger operators $i \frac{\partial}{\partial t}-H_{1}$ and $i \frac{\partial}{\partial t}-H_{2}$ such that the corresponding D-to-N operators $\Lambda_{1}$ and $\Lambda_{2}$ are gauge equivalent, i.e. $\Lambda_{1}=g_{0}^{-1} \Lambda_{2} g_{0}$ where $g_{0}=\left.g\right|_{\partial \Omega_{0} \times[0, T]}, g \in G(D)$. Denote $A^{(3)}=A^{(2)}-i g^{-1} \frac{\partial g}{\partial x}, V^{(3)}=$ $V^{(2)}+i g^{-1} \frac{\partial g}{\partial t}$. Let $i \frac{\partial}{\partial t}-H_{3}$ be the Schrödinger operator with electromagnetic potentials $A^{(3)}, V^{(3)}$. Then $i \frac{\partial}{\partial t}-H_{1}$ and $i \frac{\partial}{\partial t}-H_{3}$ have the same D-to-N operator : $\Lambda_{3}=\Lambda_{1}$.

Let $u_{1}$ be the solution of the initial-boundary value problem:

$$
\begin{array}{r}
i \frac{\partial u_{1}}{\partial t}-H_{1} u_{1}=0 \quad \text { in } D, \\
u_{1}(x, 0)=0, \quad(x, 0) \in D_{0}, \\
\left.u_{1}\right|_{\partial \Omega_{0} \times(0, T)}=f,\left.\quad u_{1}\right|_{\partial \Omega^{\prime}(t)}=0 \quad \forall t \in[0, T],
\end{array}
$$

and let $u_{3}$ be the solution of

$$
\begin{array}{r}
i \frac{\partial u_{3}}{\partial t}-H_{3}^{*} u_{3}=0 \quad \text { in } D, \\
u_{3}(x, T)=0, \quad(x, T) \in D_{T}, \\
\left.u_{3}\right|_{\partial \Omega_{0} \times(0, T)}=g,\left.\quad u_{3}\right|_{\partial \Omega^{\prime}(t)}=0 \quad \forall t \in[0, T],
\end{array}
$$

where $H_{3}^{*}$ is the adjoint to $H_{3}$.

We have, by the Green's formula (c.f. [SU]):

$$
\begin{aligned}
0 & =\left(\left(i \frac{\partial u}{\partial t}-H_{1}\right) u_{1}, u_{3}\right)-\left(u_{1},\left(i \frac{\partial}{\partial t}-H_{3}^{*}\right) u_{3}\right) \\
& =\int_{D}\left(2 i A^{(1)}(x, t) \cdot \frac{\partial u_{1}}{\partial x} \overline{u_{3}}+2 i u_{1} A^{(3)} \cdot \frac{\partial \overline{u_{3}}}{\partial x}\right. \\
+\left(q_{1}(x, t)\right. & \left.\left.-q_{3}(x, t)\right) u_{1} \overline{u_{3}}\right) d x d t+\left[\Lambda_{1} f, g\right]-\left[f, \Lambda_{3}^{*} g\right],
\end{aligned}
$$

where $q_{p}=i \frac{\partial}{\partial x} \cdot A^{(p)}(x, t)+A^{(p)}(x, t) \cdot A^{(p)}(x, t)+V^{(p)}(x, t),[$,$] is the inner$ product on $\partial \Omega_{0} \times[0, T]$ and $\Lambda_{p}$ are the D-to- $\mathrm{N}$ operators corresponding to 
$H_{p}, p=1$ or 3 . Let

$$
v=\sum_{j=0}^{r} \sum_{p=0}^{N} \frac{b_{p j}}{(i k)^{p}} e^{-i k^{2} t+i k \psi_{j}(x, t, \omega)}+v^{(N+1)}
$$

be the geometric optics solution of (2.34) corresponding to the same broken ray $\gamma$ as the solution (2.32). Substitute (2.32) in (2.35) instead of $u_{1}$ and substitute (2.36) instead of $u_{3}$. Dividing (2.35) by $2 k$, passing to the limit when $k \rightarrow \infty$ and taking into account that $\Lambda_{1}=\Lambda_{3}$ we get (c.f. [E3], pages 1502-03):

$$
\left.\sum_{j=0}^{r} \int_{D}\left(A^{(3)}(x, t)-A^{(1)}(x, t)\right) \cdot \psi_{j x}(x, t, \theta) a_{0 j}(x, t, \omega) \overline{b_{0 j}(x, t, \omega)}\right) d x d t
$$

Note that $a_{00}$ and $b_{00}$ have the form (2.4). Note also that terms containing $\psi_{j t}$ in $a_{0 j} \overline{b_{0 j}}$ cancel each other. Making changes of variables as in [E3], pages 1503-06 (see also [E4], page 30), and taking the limit when $\varepsilon \rightarrow 0$ where $\varepsilon>0$ is the same as in (2.4), we obtain

$$
\exp \left[i \sum_{j=0}^{r} \int_{\gamma_{j}}\left(A^{(3)}\left(x_{0}^{(j)}+s \theta_{j}, t_{0}\right)-A^{(1)}\left(x_{0}^{(j)}+s \theta_{j}, t_{0}\right)\right) \cdot \theta_{j} d s\right]=1,
$$

where $\theta_{j}$ is the direction of $\gamma_{j}, x_{0}^{(j)}$ is the starting point of $\gamma_{j}, 0 \leq j \leq r$.

It is important to emphasize that the ray $\gamma$ is contained in the plane $t=$ const. Therefore the tomography problem (2.37) is exactly the same as in the time-independent case.

In the case when $\gamma$ does not hit obstacles, (2.37) reduces to

$$
\exp \left\{i \int_{-\infty}^{\infty}\left[A^{(3)}\left(x_{0}^{(0)}+s \omega, t_{0}\right)-A^{(1)}\left(x_{0}^{(0)}+s \omega, t_{0}\right)\right] \cdot \omega d s\right\}=1
$$

We took in (2.38) $s_{0}=-\infty, s_{1}=+\infty$.

If conditions of Theorem 1.1 are satisfied then (2.38) implies (see [E2], $\S 2)$ that there exists $\varphi(x, t) \in C^{\infty}(\bar{D})$ such that

$$
A^{(1)}(x, t)-A^{(3)}(x, t)=\frac{\partial \varphi}{\partial x},\left.\quad \varphi\right|_{\partial \Omega_{0} \times[0, T]}=0 .
$$


If conditions of Theorem 1.2 are satisfied then (2.37) implies (see [E3], pages $1512-1515)$ that there exists $c(x, t) \in G(D)$ such that

$$
A^{(3)}-A^{(1)}=i c^{-1} \frac{\partial c}{\partial x},\left.\quad c\right|_{\partial \Omega_{0} \times[0, T]}=1 .
$$

Using (2.39) or (2.40) we make a gauge transformation $u_{4}=c^{-1} u_{3}$, where $c=e^{i \varphi}$ in the case (2.39). Then $\left(i \frac{\partial}{\partial t}-H_{4}\right) u_{4}=0$, where $i \frac{\partial}{\partial t}-H_{4}$ is the operator with electromagnetic potentials $A^{(4)}=A^{(3)}-i c^{-1} \frac{\partial c}{\partial x}, V^{(4)}=$ $V^{(3)}+i c^{-1} \frac{\partial c}{\partial t}$. Note that $A^{(1)}=A^{(4)}$. Since $c=1$ on $\partial \Omega_{0} \times(0, T)$ we have that $\Lambda_{4}=\Lambda_{3}=\Lambda_{1}$, where $\Lambda_{4}$ is the D-to- $\mathrm{N}$ operator corresponding to $i \frac{\partial}{\partial t}-H_{4}$. Apply the Green's formula (2.35) to $\left(i \frac{\partial}{\partial t}-H_{1}\right) u_{1}=0$ and $\left(i \frac{\partial}{\partial t}-H_{4}^{*}\right) u_{5}=0$, where $u_{5}(x, T)=0,\left.u_{5}\right|_{\partial \Omega_{0} \times(0, T)}=g_{1},\left.u_{5}\right|_{\partial \Omega^{\prime}(t)}=0, \forall t \in[0, T]$.

Since $A^{(1)}=A^{(4)}$ and $\Lambda_{1}=\Lambda_{4}$ we get

$$
\int_{D}\left(V^{(1)}(x, t)-V^{(3)}(x, t)-i c^{-1} \frac{\partial c}{\partial t}\right) u_{1} \overline{u_{5}} d x d t=0 .
$$

Substituting geometric optic solutions (2.32) and (2.36) with $H_{3}^{*}$ replaced by $H_{4}^{*}$ we get

$$
\sum_{j=1}^{r} \int_{\gamma_{j}}\left(V^{(1)}-V^{(3)}-i c^{-1} \frac{\partial c}{\partial t}\right) d s=0 .
$$

Note that (2.42) holds for each $t \in[0, T]$ and for each broken ray in $D_{t}$. It shown in [E2], $\S 3$, that (2.42) implies that

$$
V^{(1)}=V^{(3)}+i c^{-1} \frac{\partial c}{\partial t}
$$

Therefore (2.40), (2.43) prove Theorem 1.2.

Analogously, when $\gamma$ does not hit the obstacles we have, instead of (2.42):

$$
\int_{\gamma}\left(V^{(1)}-V^{(3)}-i c^{-1} \frac{\partial c}{\partial t}\right) d s=0 .
$$

It is shown in [E2], $\S 2$, that (2.44) also implies (2.43) with $c=e^{i \varphi(x, t)}$. Therefore Theorem 1.1 also holds.

Remark 2.2. Deriving formulas of the form (2.37) we assumed that $\gamma=\gamma_{1} \cup \ldots \cup \gamma_{r}$ are broken nontangential rays that do not have focal points 
(i.e. do not intersect the caustics set). It was noted in [E4], page 29, that these formulas still hold when $\gamma$ contains a generic caustics point.

Consider, for simplicity, the case $n=2$. Let $\left(x_{10}, x_{20}, t_{0}\right)$ be the point of the intersection of $\gamma$ with the caustics set. In the neighborhood of $\left(x_{10}, x_{20}, t_{0}\right)$ we replace the ansatz $\sum_{p=0}^{N} \frac{a_{p}(x, t)}{(i k)^{P}} e^{-i k^{2} t+i k \psi(x, t)}$ with the following ansatz (c.f. $[\mathrm{V}])$ :

$$
v=\sum_{p=0}^{N} \int_{-\infty}^{\infty} \frac{b_{p}\left(x, t, \xi_{1}\right)}{(i k)^{p-\frac{1}{2}}} e^{-i k^{2} t+i \varphi\left(x_{2}, t, \xi_{1}\right)-i x_{1} \xi_{1}} d \xi_{1} .
$$

At the caustics set we have:

$$
\varphi_{\xi_{1}}-x_{1}=0, \quad \varphi_{\xi_{1}^{2}}=0
$$

We shall assume that $\varphi_{\xi_{1}^{3}} \neq 0$ on this set. Then the following estimate holds in a neighborhood of $\left(x_{10}, x_{20}, t_{0}\right)$ :

$$
|v| \leq \frac{C k^{\frac{1}{6}}}{1+k^{\frac{1}{6}} d^{\frac{1}{4}}} \leq \frac{C}{d^{\frac{1}{4}}}
$$

where $d(x, t)$ is the distance to the caustics set. It was shown in [E4] that the estimate (2.46) leads to the proof of (2.37) and(2.42).

\section{The Schrödinger operator with time-dependent Yang-Mills potentials.}

The Schrödinger equation with time-dependent Yang-Mills potentials has the following form:

$$
L u \stackrel{\text { def }}{=} i \frac{\partial u(x, t)}{\partial t}-\sum_{j=1}^{n}\left(-i I_{m} \frac{\partial}{\partial x_{j}}-A_{j}(x, t)\right)^{2} u(x, t)+V(x, t) u(x, t)
$$

where $A_{j}, 1 \leq j \leq n, V(x, t), u(x, t)$ are $m \times m$ matrices, $I_{m}$ is the identity matrix in $\mathbf{C}^{m}$. We assume that $A_{j}, 1 \leq j \leq n, V$ are smooth in $\bar{\Omega}_{0} \times$ $[0, T]$ and without lost of generability (see Remark 3.1) we can assume that $A(x, t)=0, V(x, t)=0$ near $\partial \Omega_{0} \times(0, T)$. We also assume that $A_{j}(x, t)$ are self-adjoint matrices, $1 \leq j \leq n$. We consider (3.1) in $\Omega_{0} \times(0, T)$ with the 
initial condition (1.2) and the boundary condition $\left.u\right|_{\partial \Omega_{0} \times(0, T)}=f$. We do not consider domains with obstacles in the case of equation (3.1).

The gauge group $G$ now is the group of $m \times m$ nonsingular smooth matrices $g(x, t)$ in $\bar{\Omega}_{0} \times[0, T]$. Let $L^{(p)}$ be two Schrödinger operators of the form (3.1) with Yang-Mills potentials $A^{(p)}(x, t), V^{(p)}(x, t), p=1,2$.

Let

$$
\Lambda_{p} f_{p}=\left.\left(\frac{\partial}{\partial \nu}-i A \cdot \nu\right) u_{p}\right|_{\partial \Omega_{0} \times(0, T)}, \quad p=1,2,
$$

be corresponding D-to- $\mathrm{N}$ operators, where $L^{(p)} u_{p}=0$ in $\Omega_{0} \times(0, T), u_{p}=0$ when $t=0, p=1,2,\left.u_{p}\right|_{\partial \Omega_{0} \times(0, T)}=f_{p}$. If $u_{2}=g^{-1} u_{1}, \quad g \in G$, then

$$
\begin{array}{r}
A_{j}^{(2)}=g^{-1} A_{j}^{(1)} g+i g^{-1} \frac{\partial g}{\partial x_{j}}, \quad 1 \leq j \leq n, \\
V^{(2)}=g^{-1} V^{(1)} g-i g^{-1} \frac{\partial g}{\partial t} .
\end{array}
$$

Yang-Mills potentials $\left(A^{(2)}, V^{(2)}\right)$ and $\left(A^{(1)}, V^{(1)}\right)$ are called gauge equivalent. As in the case of Theorem 1.1 we have:

Theorem 3.1. If the D-to- $N$ operators $\Lambda_{1}$ and $\Lambda_{2}$ are gauge equivalent on $\partial \Omega_{0} \times(0, T)$ (c.f. (1.7) ) then $\left(A^{(1)}, V^{(1)}\right)$ and $\left(A^{(2)}, V^{(2)}\right)$ are gauge equivalent in $\bar{\Omega}_{0} \times[0, T]$.

The start of the proof of Theorem 3.1 is the same as of Theorem 1.1. We construct the geometric optics solution of (3.1) of the form:

$$
u_{N}=e^{-i k^{2} t+i k(x, \omega)} \sum_{p=0}^{N} \frac{1}{(i k)^{p}} a_{p}(x, t, \omega)+u^{(N+1)},
$$

where $a_{0}(x, t)=\chi_{1}(t) \chi_{2}(\tau) c(x, t, \omega)$ and

$$
\begin{array}{r}
\omega \cdot \frac{\partial c}{\partial x}-i A(x, t) \cdot \omega c(x, t)=0, \quad s>s_{0}, \\
\left.c\right|_{s=s_{0}}=I_{m},
\end{array}
$$

$a_{p}(x, t, \omega)$ are solutions of the equations $2 \omega \cdot\left(-i \frac{\partial}{\partial x}-A\right) a_{p}=L a_{p-1}$ for $s>$ $s_{0},\left.a_{p}\right|_{s=s_{0}}=0, p \geq 1$. 
Here $s, s_{0}, \chi_{1}(t), \chi_{2}(\tau)$ are the same as in (2.4), (2.5). Note that since $A(x, t)$ have a compact support we can take $s_{0}=-\infty$. Finally, $u^{(N+1)}$ satisfies

$$
\begin{array}{r}
L u^{(N+1)}=\frac{-e^{-i k^{2} t+i k(\omega \cdot x)}}{(i k)^{N}} L a_{N}, \quad(x, t) \in \Omega_{0} \times(0, T), \\
\\
u^{(N+1)}(x, 0)=0,\left.\quad u^{(N+1)}\right|_{\partial \Omega_{0}} \times(0, T)=0 .
\end{array}
$$

The existence of $u^{(N+1)}$ follows from Lemma 2.1 that holds without changes in the case of equation (3.1). Since $\Lambda_{2}=g_{0}^{-1} \Lambda_{1} g_{0}$ for some $g \in G\left(\Omega_{0} \times(0, T)\right)$ where $g_{0}=\left.g\right|_{\partial \Omega_{0} \times(0, T)}$ we have that $\Lambda_{3}=\Lambda_{1}$ where $\Lambda_{3}$ is the D-to-N operator corresponding to $L_{3}$ with potentials $A^{(3)}=g^{-1} A^{(2)} g-i g^{-1} \frac{\partial g}{\partial x}, V^{(3)}=$ $g^{-1} V^{(2)} g+i g^{-1} \frac{\partial g}{\partial t}$. Substituting the geometric optics solutions for $L_{1}$ and $L_{3}^{*}$ into the Greens formula of the form (2.35) we get as in $\S 2$ (c.f. (2.38) and also [E4], pages 29-30):

$$
c_{30}^{-1}\left(+\infty, y_{2}, t, \omega\right) c_{10}\left(+\infty, y_{2}, t, \omega\right)=I_{m},
$$

where $y_{1}=x \cdot \omega, y_{2}=x-(x \cdot \omega) \omega, c_{j}(x, t, \omega), j=1,3$, are the solution of the differential equation of the form (c.f. (3.5) ):

$$
\omega \cdot \frac{\partial c_{j}(x, t, \omega)}{\partial x}-i\left(A_{j}(x, t) \cdot \omega\right) c_{j}(x, t, \omega)=0,
$$

$c_{j 0}\left(y_{1}, y_{2}, t, \omega\right)$ is $c_{j}(x, t, \omega)$ in $\left(y_{1}, y_{2}\right)$ coordinates, $c_{j 0}\left(-\infty, y_{2}, t, \omega\right)=I_{m}$. The matrix $c_{j 0}\left(+\infty, y_{2}, t, \omega\right)$ is called the non-abelian Radon transform of $A^{(j)}(x, t), j=1,3$.

The following lemma was proven in [E5] (see also [No]):

Lemma 3.1. If the non-abelian Radon transform of $A^{(1))}$ and $A^{(3)}$ are equal then $A^{(1))}$ and $A^{(3)}$ are gauge equivalent with $g \in G_{0}\left(\Omega_{0} \times[0, T]\right.$, i.e. $g=I_{m}$ on $\partial \Omega_{0} \times[0, T]$.

The proof of Lemma 3.1 is much harder than the similar result in the abelian case (i.e. $m=1$ ), and involves a lot of the complex analysis. We consider briefly the most important case of two dimensions. The case $n \geq 3$ is reduced easily to the case $n=2$. The main ingredient of the proof is the study of an equation of the form (3.8) with complex parameters:

$$
\zeta_{1} \frac{\partial c}{\partial x_{1}}+\zeta_{2} \frac{\partial c}{\partial x_{2}}=i\left(A_{1}(x, t) \zeta_{1}+A_{2}(x, t) \zeta_{2}\right) c,
$$


where $\zeta_{1}^{2}+\zeta_{2}^{2}=1, \zeta_{i} \in \mathbf{C}, i=1,2$. Define $\zeta_{1}(\tau)=\frac{1}{2}\left(\tau+\frac{1}{\tau}\right), \zeta_{2}(\tau)=$ $\frac{i}{2}\left(\tau-\frac{1}{\tau}\right)$, where $\tau \in \mathbf{C} \backslash\{0\}$. It was proven in [ER2] that there exists solutions $c_{ \pm}(x, t, \tau)$ of (3.9) $)$, smooth in $(x, t, \tau)$ and such that $c_{+}(x, t, \tau)$ is analytic and a nonsingular matrix in $\tau$ when $|\tau|<1, c_{-}(x, t, \tau)$ is analytic and nonsingular in $\tau$ for $|\tau|>1$. Let $\tau=|\tau| e^{i \varphi}$. When $|\tau| \rightarrow 1$ we have that $\left(\zeta_{1}(\tau), \zeta_{2}(\tau) \rightarrow\right.$ $\omega(\varphi) \stackrel{\text { def }}{=}(\cos \varphi,-\sin \varphi)$. Therefore $c_{ \pm}\left(x, t, e^{i \varphi}\right)$ are solutions of equation of the form (3.8) with $\omega(\varphi)=(\cos \varphi,-\sin \varphi)$. As before, denote $y_{1}=(x \cdot \omega(\varphi))$ and $y_{2}=\left(x \cdot \omega_{\perp}(\varphi)\right)$ where $\omega_{\perp}(\varphi)=(\sin \varphi, \cos \varphi)$. Let $c_{ \pm}^{(0)}\left(y_{1}, y_{2}, t, e^{i \varphi}\right)$ be $c_{ \pm}\left(x, t, e^{i \varphi}\right)$ in $\left(y_{1}, y_{2}\right)$ coordinates and let $c_{0}\left(y_{1}, y_{2}, t, \omega(\varphi)\right)$ be the solution of the Cauchy problem (3.8). We have:

$$
c_{0}\left(y_{1}, y_{2}, t, \omega(\varphi)\right)=c_{ \pm}^{(0)}\left(y_{1}, y_{2}, t, e^{i \varphi}\right)\left(c_{ \pm}^{(0)}\left(-\infty, y_{2}, t, e^{i \varphi}\right)\right)^{-1}
$$

by the uniqueness of the Cauchy problem for (3.8). Putting $y_{1}=+\infty$ we get

$$
c_{0}\left(+\infty, y_{2}, t, \omega(\varphi)\right)=c_{ \pm}^{(0)}\left(+\infty, y_{2}, t, e^{i \varphi}\right)\left(c_{ \pm}^{(0)}\left(-\infty, y_{2}, t, e^{i \varphi}\right)\right)^{-1}
$$

It follows from (3.7) and (3.11) that

$$
\begin{aligned}
& c_{1, \pm}^{(0)}\left(+\infty, y_{2}, t, e^{i \varphi}\right)\left(c_{1, \pm}^{(0)}\left(-\infty, y_{2}, t, e^{i \varphi}\right)\right)^{-1} \\
= & c_{3, \pm}^{(0)}\left(+\infty, y_{2}, t, e^{i \varphi}\right)\left(c_{3, \pm}^{(0)}\left(-\infty, y_{2}, t, e^{i \varphi}\right)\right)^{-1},
\end{aligned}
$$

where $c_{j, \pm}\left(x, t, e^{i \varphi}\right)$ correspond to the potentials $A^{(j)}(x, t), j=1,3$. The continuation of the proof of Lemma 3.1 when $n=2$ is the same as in [E5], $\S 2$.

Now we shall consider the case $n \geq 3$ assuming that (3.7) holds. Let $\omega=\left(\omega_{1}, \ldots, \omega_{n}\right) \in S^{n-1}$ and let $\omega_{12}=\left(\omega_{1}, \omega_{2}, 0, \ldots, 0\right) \in S^{n-1}$. Consider equations (3.8) with $\omega$ replaced by $\omega_{12}$. It is a two-dimensional problem with $x_{3}, \ldots, x_{n}$ and $t$ as parameters. It follows from Lemma 3.1 for $n=2$ that there exists $g(x, t), \operatorname{det} g(x, t) \neq 0, g=I_{m}$ on $\partial \Omega_{0} \times[0, T]$ such that

$$
A_{1}^{(3)}=g^{-1} A_{1}^{(1)} g+i g^{-1} \frac{\partial g}{\partial x_{1}}, \quad A_{2}^{(3)}=g^{-1} A_{2}^{(1)} g+i g^{-1} \frac{\partial g}{\partial x_{2}}
$$

for all $(x, t)$. Replacing $\omega_{2}$ by $\omega_{j}, j>2$, we get that there exists $\tilde{g} \in$ $G_{0}\left(\Omega_{0} \times[0, T]\right)$ such that

$$
A_{1}^{(3)}=\tilde{g}^{-1} A_{1}^{(1)} \tilde{g}+i \tilde{g}^{-1} \frac{\partial \tilde{g}}{\partial x_{1}}, \quad A_{j}^{(3)}=\tilde{g}^{-1} A_{j}^{(1)} \tilde{g}+i \tilde{g}^{-1} \frac{\partial \tilde{g}}{\partial x_{2}}
$$


for all $(x, t)$. Comparing (3.13) and (3.14) we have that $g$ and $\tilde{g}$ satisfy the same system of differential equations

$$
i \frac{\partial g}{\partial x_{1}}+A_{1}^{(1)} g-g A_{1}^{(3)}=0,\left.\quad g\right|_{\partial \Omega_{0} \times[0, T]}=I_{m}
$$

for each $\left(x_{2}, \ldots, x_{n}, t\right)$.

By the uniqueness theorem for the differential equations we get that $\tilde{g}=g$. Therefore

$$
A_{j}^{(3)}=g^{-1} A_{j}^{(1)} g+i g^{-1} \frac{\partial g}{\partial x_{j}}, \quad j \geq 3
$$

Denote

$$
A^{(4)}=g A^{(3)} g^{-1}-i \frac{\partial g}{\partial x} g^{-1}, \quad V^{(4)}=g V^{(3)} g^{-1}+i \frac{\partial g}{\partial x} g^{-1}
$$

Then $A^{(4)}(x, t)=A^{(1)}(x, t)$.

Now we shall show that $V^{(4)}=V^{(1)}$, i.e. $\left(A^{(3)}, V^{(3)}\right)$ and $\left(A^{(1)}, V^{(1)}\right)$ are gauge equivalent. As before, it is enough to consider the case $n=2$. Using the Green's formula for $L^{(1)}$ and $L^{(4) *}$ we get, as in $\S 2$ (c.f. (2.41), (2.44) ), that

$$
\int_{-\infty}^{\infty} c_{10}^{-1}\left(y_{1}, y_{2}, t, \omega\right)\left(V^{(1)}(x, t)-V^{(4)}(x, t)\right) c_{10}\left(y_{1}, y_{2}, t, \omega\right) d y_{1}=0
$$

Here $y_{1}=x \cdot \omega, y_{2}=x-(x \cdot \omega) \omega$. Substituting (3.10) into (3.17, multiplying (3.16) by $c_{ \pm}^{-1}\left(-\infty, y_{2}, t, e^{i \varphi}\right)$ from the left and $c_{ \pm}\left(-\infty, y_{2}, t, e^{i \varphi}\right)$ from the right, we get

$$
\int_{-\infty}^{\infty} c_{ \pm}^{-1}\left(y_{1}, y_{2}, t, e^{i \varphi}\right)\left(V^{(1)}-V^{(4)}\right) c_{ \pm}\left(y_{1}, y_{2}, t, e^{i \varphi}\right) d y_{1}=0
$$

The continuation of proof that (3.18) leads to $V^{(1)}-V^{(4)}=0$ is the same as in [E1], pages 61-62.

Remark 3.1. In this remark we shall show that without loss of generality one can assume that $A(x, t)$ and $V(x, t)$ are smooth and equal to zero near $\partial \Omega_{0} \times[0, T]$ (c.f. [NSU], [ER1], [E1]). Let $L_{p}$ be two operators in $\Omega_{0} \times[0, T]$ such that $\Lambda_{1}=\Lambda_{2}$ on $\partial \Omega_{0} \times(0, T)$. As in [E3], pages 53-54, one can replace $\left(A^{(2)}, V^{(2)}\right)$ in $\Omega_{0} \times[0, T]$ by gauge equivalent $\left(A^{(3)}, V^{(3)}\right)$ with the gauge 
$g \in G_{0}$ such that $A^{(3)}(x, t) \cdot n(x, t)=A^{(1)}(x, t) \cdot n(x, t)$ near $\partial \Omega_{0} \times[0, T]$, where $n(x, t)$ is a smooth extension near $\partial \Omega_{0} \times[0, T]$ of the unit normal vector $n(x)$ on $\partial \Omega_{0}$. We shall show that $\Lambda_{1}=\Lambda_{3}$ on $\partial \Omega_{0} \times(0, T)$ implies that $A^{(1)}=A^{(3)}$ and $V^{(1)}=V^{(3)}$ on $\partial \Omega_{0} \times[0, T]$ with its derivatives. Introduce in a neighborhood $U$ of an arbitrary point $\left(x_{0}, t_{0}\right) \in \partial \Omega_{0} \times\left[0, T_{0}\right]$ a system of coordinates $(y, t)$ such that the equation of $\partial \Omega_{0}$ is $y_{n}=0$. Let $\left(\eta_{1}, \ldots, \eta_{n}, \eta_{0}\right)$ be the dual coordinates to $(y, t)$ in the cotangent space $T^{*}(U \times[0, T))$. The Schrödinger operators $L_{1}$ and $L_{3}$ are elliptic microlocally in the region where $\left|\eta_{0}\right|<\varepsilon \sum_{k=1}^{n} \eta_{k}^{2}$ (c.f. [E6], Remark 2.2). Therefore the elliptic parametrix for $\Lambda_{1}$ and $\Lambda_{3}$ applies in this region. Therefore, as in [E1], Proposition 2.1, we get that

$$
\frac{\partial^{p+q} A^{(1)}}{\partial x^{p} \partial t^{q}}=\frac{\partial^{p+q} A^{(3)}}{\partial x^{p} \partial t^{q}} \quad \text { and } \quad \frac{\partial^{p+q} V^{(1)}}{\partial x^{p} \partial t^{q}}=\frac{\partial^{p+q} V^{(3)}}{\partial x^{p} \partial t^{q}}
$$

on $\partial \Omega_{0} \times(0, T)$ for any $p$ and $q$. Therefore we can extend smoothly $\left(A^{(1)}, V^{(1)}\right)$ and $\left(A^{(3)}, V^{(3)}\right)$ from $\bar{\Omega}_{0} \times[0, T]$ to a large domain $\bar{\Omega}_{0} \times[0, T]$ in a such way that $A^{(1)}=A^{(3)}, V^{(1)}=V^{(3)}$ in $\left(\tilde{\Omega}_{0} \backslash \Omega_{0}\right) \times[0, T]$ and $\left(A^{(1)}, V^{(1)}\right)=\left(A^{(3)}, V^{(3)}\right)=$ $(0,0)$ near $\partial \tilde{\Omega}_{0} \times[0, T]$. It remains to show that $\Lambda_{1}=\Lambda_{3}$ on $\partial \Omega_{0} \times(0, T)$ implies that $\Lambda_{1}=\Lambda_{3}$ on $\partial \tilde{\Omega}_{0} \times[0, T]$. Take any smooth $f$ on $\partial \tilde{\Omega}_{0} \times[0, T]$ and consider the initial-boundary value problem

$$
\begin{array}{r}
L^{(1)} u_{1}=0 \quad \text { in } \tilde{\Omega}_{0} \times(0, T), \\
u_{1}(x, 0)=0,\left.\quad u_{1}\right|_{\partial \tilde{\Omega}_{0} \times[0, T]}=f
\end{array}
$$

Let $f_{1}$ be the restriction of $u_{1}$ to $\partial \Omega_{0} \times(0, T)$. Consider the initial-boundary value problem

$$
\begin{array}{r}
L_{3} u_{3}=0 \quad \text { in } \Omega_{0} \times(0, T), \\
u_{3}(x, 0)=0,\left.\quad u_{3}\right|_{\partial \Omega_{0} \times[0, T]}=f_{1} .
\end{array}
$$

Let $w_{3}=u_{1}$ in $\left(\bar{\Omega}_{0} \backslash \Omega_{0}\right) \times(0, T), w_{3}=u_{3}$ in $\Omega_{0} \times(0, T)$. Since $\Lambda_{1}=\Lambda_{3}$ on $\partial \Omega_{0} \times(0, T)$ we get that $w_{3}$ is the solution of $L_{3} w=0$ in $\tilde{\Omega}_{0} \times(0, T)$. Moreover, $w_{3}(x, 0)=0, x \in \tilde{\Omega}_{0}$ and $\left.w_{3}\right|_{\partial \tilde{\Omega} \times(0, T)}=f$. Since $L^{(1)}=L^{(3)}$ and $u_{1}=w_{3}$ in $\left(\tilde{\Omega}_{0} \backslash \Omega_{0}\right) \times(0, T)$ we have that $\Lambda_{1}=\Lambda_{3}$ on $\partial \tilde{\Omega}_{0} \times(0, T)$. Therefore we have reduced the inversee problem in $\Omega_{0} \times(0, T)$ to an equivalent inverse problem in a larger domain $\tilde{\Omega}_{0} \times(0, T)$. Note that the same proof works when $\Omega_{0} \times(0, T)$ contains obstacles. 


\section{Examples of the gauge equivalence classses and the Aharonov-Bohm effect.}

The results of $\S 2$ are valid in the multiconnected domains $D$. In such domains there are electromagnetic potentials $(A(x, t), V(x, t))$ and $\left(A^{\prime}(x, t), V^{\prime}(x, t)\right)$ that correspond to the same electromagnetic fields in $D: \operatorname{curl} A=\operatorname{curl} A^{\prime}=$ $B, E=-\frac{\partial V}{\partial x}-\frac{\partial A}{\partial t}=-\frac{\partial V^{\prime}}{\partial x}-\frac{\partial A^{\prime}}{\partial t}$, however $(A, V)$ and $\left(A^{\prime}, V^{\prime}\right)$ are not gauge equivalent. There is a simple description of all gauge equivalent classes of potentials in $D$ (c.f. [WY], [OP], [Va]):

Fix a point $\left(x_{0}, t_{0}\right) \in \partial \Omega_{0} \times[0, T]$ and denote by $\mathcal{P}$ the group of all paths $\gamma$ in $\bar{D}$ that start and end at $\left(x_{0}, t_{0}\right)$. Let $\int_{\gamma} A(x, t) \cdot d x-V(x, t) d t$ be a line integral in $D$. Denote

$$
R(A, V, \gamma)=\exp \left\{i\left(\int_{\gamma}-A(x, t) \cdot d x+V(x, t) d t\right)\right\} .
$$

$R$ is called the nonintegrable phase factor (c.f. [WY], [OP]). $R\left(A, V, \gamma_{0}\right)$ is well-defined for any closed path $\gamma_{0}$ in $\bar{D}$ : Let $\gamma_{1}$ be a path connecting $\left(x_{0}, t_{0}\right)$ with an arbitrary point $\left(x_{1}, t_{1}\right) \in \gamma_{0}$ and let $\gamma \in \mathcal{P}$ be a close path $\gamma_{1} \cup \gamma_{0}$. It is obvious that $R(A, V, \gamma)=R\left(A, V, \gamma_{0}\right)$. If $(A, V)$ and $\left(A^{\prime}, V^{\prime}\right)$ are gauge equivalent then

$$
\begin{array}{r}
R^{\prime} R^{-1}=\exp \left\{i\left(\int_{\gamma}\left(A-A^{\prime}\right) \cdot d x+\left(V^{\prime}-V\right) d t\right)\right\} \\
=\exp \left\{-i\left(\int_{\gamma} i c^{-1} \frac{\partial c}{\partial x} \cdot d x+i c^{-1} \frac{\partial c}{\partial t} d t\right)\right\}=\exp \left\{\int_{\gamma} d(\log c)\right\}=1,
\end{array}
$$

since $c(x, t) \in G(D)$ is a single-valued in $\bar{D}$. Therefore $R^{\prime}=R$. And vice versa, if $R(A, V, \gamma)=R\left(A^{\prime}, V^{\prime}, \gamma\right)$ for all $\gamma \in \mathcal{P}$ then $(A, V)$ and $\left(A^{\prime}, V^{\prime}\right)$ are gauge equivalent : Let

$$
\exp \left\{i\left(\int_{\gamma}\left(A^{\prime}-A\right) \cdot d x+\left(V-V^{\prime}\right) d t\right)\right\}=1
$$

for all $\gamma \in \mathcal{P}$. Let $(x, t)$ be an arbitrary point in $\bar{D}$. Denote by $c(x, t)$ the integral

$$
c(x, t)=\exp \left\{i\left(\int_{\gamma(x, t)}\left(A^{\prime}-A\right) \cdot d x+\left(V-V^{\prime}\right) d t\right)\right\}
$$


where $\gamma(x, t)$ is an arbitrary path in $\bar{D}$ connecting $\left(x_{0}, t_{0}\right)$ and $(x, t)$. It follows from (4.2) that (4.3) does not depend on $\gamma(x, t)$ connecting $\left(x_{0}, t_{0}\right)$ and $(x, t)$. Differentiating (4.3) in $x$ and $t$ we get that $(A, V)$ and $\left(A^{\prime}, V^{\prime}\right)$ are gauge equivalent.

For fixed $(A, V) R(A, V, \gamma)$ defines a map of the group of paths $\mathcal{P}$ to the group $U$ of complex numbers $z$ with absolute value 1 , i.e. $z=e^{i \alpha}, \alpha \in \mathbf{R}$ (we assume in this section that $(A, V)$ are real-valued). The image of this map $U(A, V)$ is a subgroup of $U$ and it is called the monodromy group of the connection $\mathcal{A}=\sum_{j=1}^{n} A_{j} d x_{j}-V d t$ (c.f. [Va]). We will often write $\mathcal{A}$ instead of $(A, V)$. Therefore there is one-to-one correspondence between the gauge equivalence classes of electromagnetic potentials and the monodomy groups. It was shown by Aharonov and Bohm (c.f. [AB]) that potentials belonging to different gauge equivalence classes produce a different physical impact and it can be detected in experiments. This phenomenon is called the Aharonov-Bohm effect.

Let $S$ be a two-dimensional smooth surface in $D$ such that $\partial S=\gamma$. It is convenient to represent the electromagnetic field $(E, B)$ as a two-form $\mathcal{F}=\sum_{1<j<k<n} B_{j k} d x_{j} \wedge d x_{k}+\sum_{k=1}^{n} E_{k} d x_{k} \wedge d t$, where $B_{j k}=-B_{k j}$. Note that $\mathcal{F}=d \mathcal{A}$. Therefore $B_{k j}=\frac{\partial A_{j}}{\partial x_{k}}-\frac{\partial A_{k}}{\partial x_{j}}, E_{j}=-\frac{\partial A_{j}}{\partial t}-\frac{\partial V}{\partial x_{j}}, 1 \leq j \leq n, 1 \leq$ $k \leq n$. When $n=3$ we have $B=\left(B_{1}, B_{2}, B_{3}\right), E=\left(E_{1}, E_{2}, E_{3}\right), B_{1}=$ $B_{23}, B_{2}=-B_{13}, B_{3}=B_{12}$. Therefore, when $n=3$

$$
B=\operatorname{curl} A, \quad E=-\frac{\partial A}{\partial t}-\frac{\partial V}{\partial x}
$$

Using the Stoke's formula $\int_{\gamma} \mathcal{A}=\int_{S} d \mathcal{A}$ we get (c.f. [OP]):

$$
\begin{array}{r}
\int_{\gamma} A \cdot d x-V d t=\int_{S}\left(B_{1} d x_{2} d x_{3}+B_{2} d x_{1} d x_{3}+B_{3} d x_{1} d x_{2}\right. \\
\left.+E_{1} d t d x_{1}+E_{2} d t d x_{2}+E_{3} d t d x_{3}\right) .
\end{array}
$$

The right hand side of (4.5) is called the electromagnetic flux. Note that the electromagnetic flux does not depend on the surface $S$ such that $\partial S=\gamma$. This follows from the three-dimensional Stoke's theorem and the equality $d \mathcal{F}=d(d \mathcal{A})=0$.

In the case $n=2$ formula (4.5) for the electromagnetic flux has the form:

$$
\int_{\gamma} A_{1} d x_{1}+A_{2} d x_{2}-V d t=\int_{S}\left(B_{3} d x_{1} d x_{2}+E_{1} d t d x_{1}+E_{2} d t d x_{2}\right) .
$$


Formulas (4.5), (4.6) can be used for the computations of $R(A, V, \gamma)$, especially when either the magnetic or electric fields is "shielded" inside the obstacles, i.e. when either $B$ or $E$ is nonzero in $\Omega^{\prime}(t)=\cup_{j=1}^{m} \Omega_{j}(t)$ and zero in $D$.

The following examples give a description of gauge equivalence classes of electromagnetic potentials using the electromagnetic fluxes.

Consider first an example when electromagnetic field is time-independent. Let $n=3, D_{0}=\{x:|x|<r\}$ and the single obstacle $D_{1}$ is a torus inside $D_{0}$ as in Remark 3.3 in [E3]. Let $B(x)$ be a magnetic field in $D_{0}$ such that supp $B \subset D_{1}$, i.e. $B$ is "shielded" in $D_{1}$. Denote by $b_{0}$ the flux of $B$ over arbitrary cross-section $S_{0}$ of the torus $D_{1}$.

Note that $b_{0}$ does not depend on the choice of the cross-section since $\operatorname{div} B=0$. Let $A$ be the magnetic potential in $D_{0}$, i.e. $\operatorname{curl} A=B$ in $D_{0}$. Since $B=0$ in $D_{0} \backslash D_{1}$ the integral $\int_{\gamma} A \cdot d x$ depends only on the homotopy class of $\gamma$ in $D_{0} \backslash D_{1}$. If $B^{\prime}$ is another magnetic field in $D_{0}$ shielded in $D_{1}$ and curl $A^{\prime}=B^{\prime}$ in $D_{0}$ then potentials $(A, V)$ and $\left(A^{\prime}, V^{\prime}\right)$ are gauge equivalent in $D_{0} \backslash D_{1}$ iff $V^{\prime}=V$ and there exists $m_{0} \in \mathbf{Z}$ such that the flux $\int_{S_{0}}\left(B^{\prime} \cdot n\right) d s=b_{0}+2 \pi m_{0}$, where $n$ is the unit vector normal to $S_{0}$.

In the case of time-dependent electromagnetic potential the situation is more complicated because both the magnetic and the electric field can not be shielded inside the obstacle. Moreover, we have to use the flux (4.5) instead of the magnetic flux only.

Consider the case $n=2, \Omega_{0}=\{x:|x|<r\}$ with an obstacle $\Omega_{1}$ moving with speed $v_{0}$ in the direction of $x_{1}$-axis, $\Omega_{1}=\cup_{t \in(0, T)} \Omega_{1}(t)$, where $\Omega_{1}(t)=$ $\left.\left\{(x, t):\left(x_{1}-v_{0} t\right)^{2}+x_{2}^{2} \leq r_{1}\right\}, t \in[0, T]\right\}, r_{1}<r$ is small. Here $D=$ $\left(\Omega_{0} \times(0, T)\right) \backslash \Omega_{1}$. In this case $G(D)$ consists of the functions $c(x, t)$ having the form

$$
c(x, t)=e^{i m \theta+i \psi(x, t)},
$$

where $m \in \mathbf{Z}, \psi(x, t) \in C^{\infty}(\bar{D})$ and $\theta$ is the angle in polar coordinates centered at $\left(v_{0} t, 0\right)$ with $x_{2}=0, x_{1}-v_{0} t>0$ as the polar axis. Consider an electric field $E$ "shielded" by the "moving" obstacle $\Omega_{1}$. Suppose $E=\left(0, E_{2}\right)$ where

$$
E_{2}(x, t)=\delta\left(x_{1}-v_{0} t\right) \delta\left(x_{2}\right) e(t)
$$

$e(t) \in C^{\infty}, e(t)=$ const when $t \notin\left[t_{1}, t_{1}+\varepsilon\right]$, where $t_{1}>0, t_{1}+\varepsilon<T$. Let $\Omega_{1}\left(x_{10}\right)$ be the intersection of the plane $x_{1}=x_{10}$ with $\Omega_{1}$ and let $\gamma\left(x_{10}\right)$ 
be a simple close curve in $\left(\Omega_{0} \times(0, T)\right) \cap\left\{x_{1}=x_{10}\right\}$ encircling $\Omega_{1}\left(x_{10}\right)$. By formula (4.6) we have

$$
\int_{\gamma\left(x_{10}\right)} A_{2} d x_{2}-V d t=\int_{\Omega_{1}\left(x_{10}\right)} E_{2} d x_{2} d t=\frac{1}{v_{0}} e\left(\frac{x_{10}}{v_{0}}\right) .
$$

The right hand side of (4.9) is the electric flux over $\Omega_{1}\left(x_{10}\right)$ and $V(x, t)$ is an electric potential corresponding to $E=\left(0, E_{2}\right)$. Note that (4.9) holds with $e(t)$ replaced by 0 for any closed Jordan curve in $\left\{x_{1}=x_{10}\right\} \backslash \Omega_{1}$. Let $B_{3}(x, t)$ be the magnetic field caused by the electric field $\left(0, E_{2}\right)$. It follows from the Maxwell's equations that

$$
\frac{\partial E_{2}}{\partial x_{1}}=\frac{\partial B_{3}}{\partial t}
$$

We get from (4.8)

$$
B_{3}(x, t)=-\frac{1}{v_{0}} \delta\left(x_{1}-v_{0} t\right) \delta\left(x_{2}\right) e(t)-\frac{1}{v_{0}^{2}} \theta\left(x_{1}-v_{0} t\right) \delta\left(x_{2}\right) \frac{\partial e\left(\frac{x_{1}}{v_{0}}\right)}{\partial t},
$$

where $\theta(\tau)=1$ when $\tau>0, \theta(\tau)=0$ when $\tau<0$.

Let $\gamma(t)$ be a closed simple curve in $\left(\Omega_{0} \cap\left\{t_{1}=\right.\right.$ const $\left.\}\right) \backslash \Omega_{1}(t)$. We have by $(4.6)$

$\int_{\gamma(t)} A \cdot d x=\int_{S(t)} B_{3} d x_{1} d x_{2}=-\frac{1}{v_{0}} e(t)-\frac{1}{v_{0}^{2}} \int_{S(t)} \theta_{1}\left(x_{1}-v_{0} t\right) \delta\left(x_{2}\right) \frac{\partial e\left(\frac{x_{1}}{v_{0}}\right)}{\partial t} d x_{1} d x_{2}$,

where $\partial S(t)=\gamma(t)$. Since $\frac{\partial}{\partial t} e\left(\frac{x_{1}}{v_{0}}\right)=v_{0} \frac{\partial}{\partial x_{1}} e\left(\frac{x_{1}}{v_{0}}\right)$ we get

$$
\int_{S(t)} \theta\left(x_{1}-v_{0} t\right) \delta\left(x_{2}\right) \frac{\partial e\left(\frac{x_{1}}{v_{0}}\right)}{\partial t} d x_{1} d_{2}=v_{0}\left(e\left(\frac{\tilde{x}_{1}(t)}{v_{0}}\right)-e(t)\right)
$$

where $\tilde{x}_{1}(t)$ is the intersection of the line $x_{1}=v_{0} t, x_{2}=0$ with $\gamma(t)$. Therefore

$$
\int_{S(t)} B_{3} d x_{1} d x_{2}=-\frac{1}{v_{0}} e\left(\frac{\tilde{x}_{1}(t)}{v_{0}}\right)
$$

Note that knowing the electric flux (4.9) for all $x_{10}$ we can determine the magnetic flux (4.11). Let $E_{2}^{\prime}(x, t)=\delta\left(x_{1}-v_{0} t\right) \delta\left(x_{2}\right) e^{\prime}(t)$ be shielded by the obstacle $\Omega_{1}$, where $e^{\prime}(t)=$ const when $t \notin\left[t_{1}, t_{1}+\varepsilon\right]$, and let $B_{3}^{\prime}$ be the 
coresponding magnetic field. Let $(A, V)$ and $\left(A^{\prime}, V^{\prime}\right)$ be any corresponding electromagnetic potentials in $\Omega_{0} \times(0, T)$. If $(A, V)$ and $\left(A^{\prime}, V^{\prime}\right)$ are gauge equivalent in $D$ then there exists $m \in \mathbf{Z}$ such that

$$
\frac{1}{v_{0}} e^{\prime}\left(\frac{x_{1}}{v_{0}}\right)=\int_{\Omega_{1}\left(x_{1}\right)} E_{2}^{\prime} d x_{2} d t=\int_{\Omega_{1}\left(x_{1}\right)} E_{2} d x_{2} d t+2 \pi m=\frac{1}{v_{0}} e\left(\frac{x_{1}}{v_{0}}\right)+2 \pi m
$$

for all $x_{1}$. Vice versa, suppose (4.12) holds. Then for the magnetic fluxes we also have

$$
\int_{S(t)} B_{3}^{\prime} d x_{1} d x_{2}=\int_{S(t)} B_{3} d x_{1} d x_{2}+2 \pi m
$$

for any $S(t)$ such that $\gamma(t)=\partial S(t)$ is simple closed curve in $\Omega_{0} \backslash \Omega_{1}(t)$. Moreover, (4.10) and (4.12) imply that $B_{3}-B_{3}^{\prime}=0$ in $\Omega_{0} \backslash \Omega_{1}(t)$ for all $t$. This means that $B_{3}-B_{3}^{\prime}$ as $E_{2}-E_{2}^{\prime}$ are confined to the obstacle $\Omega_{1}$. Therefore for any close path $\gamma$ in $\left(\Omega_{0} \times(0, T)\right) \backslash \Omega_{1}$ the line integral $\int_{\gamma}\left(A^{\prime}-A\right) \cdot d x+\left(V-V^{\prime}\right) d t$ depends only on the homotopy class of $\gamma$. Making a homotopy of $\gamma$ to a multiple of the $\gamma\left(x_{10}\right)$ we get, using (4.12), that $\int_{\gamma}\left(A^{\prime}-A\right) \cdot d x+\left(V-V^{\prime}\right) d t=$ $2 \pi m^{\prime}$, where $m^{\prime} \in \mathbf{Z}$. Therefore $(A, V)$ and $\left(A^{\prime}, V^{\prime}\right)$ are gauge equialent.

Finally, let $n=2$ and $\Omega_{0}, \Omega_{1}$ be the same as above. Consider an example of the electromagnetic fluxes caused by the magnetic field $B_{3}(x, t)=\delta\left(x_{1}-\right.$ $\left.v_{0} t\right) \delta\left(x_{2}\right) b(t)$ in $\Omega_{0} \times(0, T)$ shielded in $\Omega_{1}$. In this case we can allow $v_{0}=0$, i.e. the obstacle $\Omega_{1}$ may not move. Let $\gamma\left(t_{0}\right)$ be a closed simple curve in $\Omega_{0} \times\left\{t=t_{0}\right\}$ that encircles $\Omega_{1}\left(t_{0}\right)$. Then by (4.6)

$$
\int_{\gamma\left(t_{0}\right)} A \cdot d x=\int_{\Omega_{1}\left(t_{0}\right)} B_{3} d x_{1} d x_{2}=b\left(t_{0}\right) .
$$

Here $A(x, t)$ are arbitrary magnetic potentials in $\Omega_{0} \times(0, T)$ such that curl $A=$ $B(x, t)$. For example, we may take

$$
A=\left(A_{1}(x), A_{2}(x)\right)=\frac{b(t)}{2 \pi}\left(\frac{-x_{2}}{\left(x_{1}-v_{0} t\right)^{2}+x_{2}^{2}}, \frac{x_{1}-v_{0} t}{\left(x_{1}-v_{0} t\right)^{2}+x_{2}^{2}}\right) .
$$

Let $B_{3}^{\prime}(x, t)$ be an arbitrary magnetic field shielded in $\Omega_{1}$ and such that

$$
\int_{\Omega_{1}(t)} B_{3}^{\prime}(x, t) d x_{1} d x_{2}=b(t)+2 \pi m
$$

for some $m \in \mathbf{Z}$. Denote by $A^{\prime}$ an arbitrary magnetic potential in $\Omega_{0} \times(0, T)$ such that curl $A^{\prime}=B_{3}^{\prime}$ in $\Omega_{0} \times(0, T)$. Then we have

$$
\exp \left(i \int_{\gamma(t)} A \cdot d x\right)=\exp \left(i \int_{\gamma\left(t_{0}\right)} A^{\prime} \cdot d x\right)
$$


for any closed path in $\left(\Omega_{0} \times\left\{t=t_{0}\right\}\right) \backslash \Omega_{1}\left(t_{0}\right), \forall t_{0} \in[0, T]$. Therefore there exists $c(x, t) \in G(D)$ such that $A^{\prime}-A=-i c^{-1} \frac{\partial c}{\partial x}$ in $D$.

Define $V^{\prime}=V+i c^{-1} \frac{\partial c}{\partial t}$. Then $\left(A^{\prime}, V^{\prime}\right)$ is gauge equivalent to $(A, V)$. Vice versa, if $\left(A^{\prime}, V^{\prime}\right)$ and $(A, V)$ are gauge equivalent then (4.13) holds.

Note that in this example the magnetic field is shielded in $\Omega_{1}$ but the electric field $E=-\frac{\partial V}{\partial x}-\frac{\partial A}{\partial t}$ is not shielded in $\Omega_{1}$. In the previous example the electric field $E$ was shielded in $\Omega_{1}$ but the magnetic field was not.

\section{References}

[AB] Aharonov, Y. and Bohm, D., Significance of electromagnetic potentials in quantum theory, Phys.Rev., Second Series 115, 485-491 (1959)

[B] Belishev, M., 1997, Boundary control in reconstruction of manifolds and metrics (the BC method), Inverse Problems 13, R1-R45

[E1] Eskin, G., 2003, Inverse boundary value problems and the AharonovBohm effect, Inverse Problems 19, 49-62

[E2] Eskin, G., 2003, Inverse problems for the Schrödinger operators with electromagnetic potentials in domains with obstacles, Inverse Problems 19, 985-998

[E3] Eskin, G., 2004, Inverse boundary value problems in domains with several obstacles, Inverse Problems 20, 1497-1516

[E4] Eskin, G., 2005, Inverse problems for Schrödinger equations with Yang-Mills potentials in domains with obstacles and the AharonovBohm effect, Journal of Physics : Conference series 12, 23-31

[E5] Eskin, G., 2004, On non-abelian Radon transform, Russian Journ. of Math. Phys. 11, 391-408

[E6] Eskin, G., 2006, A new approach to the hyperbolic inverse problems, Inverse Problems 22, 815-831

[ER1] Eskin, G. and Ralston, J., (1997), Inverse scattering problems for Schrödinger operators with magnetic and electric potentials, IMA, vol. Math. Appl. 90, 147-166 
[ER2] Eskin, G. and Ralston, J., (2004), On the inverse boundary value problems for the linear isotropic elasticity and Cauchy-Riemann systems, Contemp. Math. 348, 53-69

[KKL] Katchalov, A., Kurylev, Y., Lassas, M., 2001, Inverse boundary spectral problems (Boca Baton: Chapman\&Hall)

[N] Nicoleau, F., An inverse scattering problem with the Aharonov-Bohm effect, Journ. Math. Phys., 41, 5223-5237 (2000)

[No] Novikov, R., (2002), On determination of a gauge field on $\mathbf{R}^{d}$ from its non-Abelian Radon transform along oriented straight lines, J. Inst. Math. Jussieu 1, 559-629

[NSU] Nakamura, G., Sun, Z., Uhlmann, G., Global identifiability for inverse problem for the Schrödinger equation in a magnetic field, Math.Ann. $303,377-88$

[OP] Olarin, S. and I. Iovitzu Popescu, 1985, The quantum effects of electromagnetic fluxes, Review of Modern Physics, vol. 57, N2, 339-436

[SU] Sylvester, J. and Uhlmann, G., 1987, A global uniqueness theorem for an inverse boundary value problem, Ann. Math. 125, 153-69

[V] Vainberg, B., 1989, Asymptotic methods in equations of mathematical physics (New York: Gordon and Breach)

[Va] Varadarajan, V.S., 2003, Vector bundles and connections in physics and mathematics: some historical remarks, Trends Math, 502-541, Birkhauser, Basel

[W] Weder, R. Aharonov-Bohm effect and time-dependent inverse scattering theory, preprint (2001)

[WY] Wu, T. and Yang, C., Phys. Rev. D 12,3845 (1975) 